\title{
Comparison of a Hearing Aid Fitting Formula Based on Korean Acoustic Characteristics and Existing Fitting Formulae
}

\author{
In-Ki Jin', Kyoungwon Lee², Jinsook Kim', Dongwook Kim³, Junil Sohn³, Junghak Lee ${ }^{2}$ \\ 'Division of Speech Pathology and Audiology, Research Institute of Audiology and Speech Pathology, College of Natural Sciences, Hallym University, \\ Chuncheon, Korea \\ ${ }^{2}$ Department of Audiology, Hallym University of Graduate Studies, Seoul, Korea \\ ${ }^{3}$ Future IT Research Center, Samsung Advanced Institute of Technology, Yongin, Korea
}

한국어의 음향 특성을 고려한 보청기 처방법과 기존 처방법의 비교

진인기 ${ }^{1} \cdot$ 이경원 ${ }^{2} \cdot$ 김진숙 ${ }^{1} \cdot$ 김동욱 ${ }^{3} \cdot$ 손준일 $^{3} \cdot$ 이정학 $^{2}$

한림대학교 자연과학대학 언어청각학부 · 청각언어연구소 ${ }^{1}$, 한림국제대학원대학교 청각학과 ${ }^{2}$, 삼성종합기술원 미래IT연구센터 ${ }^{3}$

\begin{abstract}
Purpose: Most existing hearing aid (HA) fitting formulae were developed based on English. However, Korean language has different acoustic characteristics compared to English. This study aimed to develop Korean language-specific hearing aid fitting formula (Hallym Audiology Institute-version 1, HAl-v1) and to compare speech perception, clarity, and overall preference between the HAl-v1 and established fitting formulae [National Acoustic Laboratories' nonlinear fitting procedure, version 2 (NAL-NL2) and Desired Sensation Level, version 5.0 (DSL5.0)]. Methods: Gains for the HAl-v1 were determined to reach the gain around the medium to the two-thirds portion in the dynamic range of conversational speech level for soft, medium, and loud levels using loudness growth for Koreans who have sensorineural hearing loss (SNHL). Twenty-four people with SNHL participated in this study. Reception thresholds of sentences (RTSs) and preferences (clarity and overall preference) were evaluated, once with HAI-v1 setting, once with NAL-NL2 setting, and once with DSL5.0 setting, in random order. Then, results of RTSs and preferences across HA fitting formulae were compared. Results: There were no statistical differences for RTSs with and without noise in all HA fitting formulae. For clarity, more participants preferred sounds with HAI-v1 or NAL-NL2 than sounds with DSL5.0. For overall preference, more participants preferred sounds with HAI-v1 than sounds with NAL-NL2 or DSL5.0. Conclusion: Results of this study suggest that language-specific HA fitting formula may provide better HA outcomes for people who use different languages.
\end{abstract}

Key Words: Hearing aid fitting formula, Hallym Audiology Institute-version 1, Speech perception, Quality judgment.

Received: April 4, 2017 / Revised: June 23, 2017 / Accepted: June 25, 2017

Correspondence: Junghak Lee, Department of Audiology, Hallym University of Graduate Studies, 427 Yeoksam-ro, Gangnam-gu, Seoul 06197, Korea Tel: +82-2-2051-4950 / Fax: +82-2-3453-6618 / E-mail: leejh@hallym.ac.kr

\section{INTRODUCTION}

Generally, fittings for modern hearing aids (HAs) follow two steps. The first step is an initial fitting, which is based on HA fitting formulae such as [National Acoustic Laboratories' nonlinear fitting procedure, version 2 (NAL-NL2) and Desired Sensation Level, version 5.0 (DSL5.0)] (Keidser et al., 2012; Scollie et al., 2005). The target of the initial fitting is to provide proper gains across frequencies and is based on general characteristics for peo- ple who have hearing loss (Keidser et al., 2012; Scollie et al., 2005). The second step is a fine adjustment, which is called fine tuning. The target of fine tuning is to fit the HA based on individual hearing and cognitive characteristics and preferences (Mueller et al., 1992). Although fine tuning is important for successful outcome of individual HA users, an accurate initial fitting is necessary for efficient fine tuning (Keidser et al., 2012; Mueller et al., 1992; Scollie et al., 2005).

For the initial fitting, there are several widely used non-linear 
HA fitting formulae such as the NAL-NL2 and the DSL5.0 (Keidser et al., 2012; Scollie et al., 2005). NAL-NL2 was developed to make loudness comfortable while maximizing speech intelligibility (Keidser et al., 2012). DSL5.0 was developed to provide sufficient audibility of important acoustic cues as much as possible (Scollie et al., 2005). Although these formulae have different rationales, restoring audibility is a key purpose for both formulae which are mainly based on English (Keidser et al., 2012; Scollie et al., 2005).

This study considered a Korean language-specific HA fitting formula. Most of HA fitting formulae were developed to provide optimized individuals' audibility (Keidser et al., 2012; Scollie et al., 2005). To restore audibility for people who have hearing loss, acoustic and linguistic characteristics of the language such as long-term average speech spectrum (LTASS), dynamic range (DR) of speech, and band-importance function (BIF) should be considered because these characteristics contribute to measure the degree of necessary amplification across frequency bands (Palmer \& Lindley, 2002). However, such characteristics differ between Korean and English. For example, Noh \& Lee (2012) reported that Korean language showed different LTASS compared to English. Specifically, high frequency energy for Korean LTASS was less than the energy for English LTASS. In addition, the DR of Korean speech differs from the DR of English (Jin et al., 2014). Jin et al. (2014) reported that the DR for Korean was narrower than the English DR in low-frequencies. Moreover, Korean BIFs were differed from the English BIFs (Jin et al., 2016). BIF represents a relative contributions of speech intelligibility to different frequency areas. In the study, band-importance weights for the Korean BIF were more important compared to the English BIF. Results of previous studies indicate that the Korean languagespecific HA fitting formula may provide better audibility for Korean people who have hearing loss compared to formulae based on other languages like English.

The purpose of this study was to develop Korean languagespecific HA fitting formula (Hallym Audiology Institute-version 1, HAI-vl) and to compare differences in speech perception and preferences across HA fitting formulae of HAI-vl, NAL-NL2, and DSL5.0. First, Gains for the HAI-v1 were determined to reach the gain around the medium to the two-thirds portion in the dynamic range of conversational speech level (CSL) for soft, medium, and loud levels using loudness growth for Koreans who have sensorineural hearing loss (SNHL). Second, results of speech perception and preferences across different HA fitting formulae were compared. This study will provide information about whether HA fitting formulae need to be specific to the native language of the HA user.

\section{MATERIALS AND METHODS}

\section{Derivation of the HA fitting formula for Korean (HAl-v1)}

Because many variables for HA and patient condition affect HA outcomes, the current study considered only one specific condition including adults with SNHL, monaural, experienced HA user, behind-the-ear (BTE) HA, 2 cc coupler, 8 channels, ventoccluded, and tube no. 13 when insertion gains were determined for each frequency of the HAI-vl.

The rationale of the HAI-v1 was to make loudness comfortable while providing sufficient audibility for low [ $40 \mathrm{~dB}$ sound pressure level (SPL)], medium (60 dB SPL), and high level (80 dB SPL) sounds. For the loudness, gains were determined to follow the normal loudness growth. Thus, reported data of loudness growth for Koreans who have SNHL (Park \& Lee, 2013) was used for determining the most comfortable level (MCL) and uncomfortable level (UCL) across frequencies. The MCL and UCL values were calculated based on a patient's hearing threshold in a given frequency band and a frequency-dependent weighted factor (Park \& Lee, 2013).

For audibility for low, medium, and high levels of sound, CSLs were measured. Three male and three female speakers (22-26 years) who had normal hearing $(<20 \mathrm{~dB} H \mathrm{HL})$ and had no articulation problems participated in the recording of the CSL. The Korean standard sentence lists for adults (KS-SL-A) (Jang et al., 2008) with efforts of soft, medium, and loud levels were recorded by speakers. The average soft speech level (LZeq) was 56.7 $\mathrm{dB}$ [standard deviation $(\mathrm{SD}): \pm 3.5 \mathrm{~dB}$ ], the medium speech level was $63.0 \mathrm{~dB}$ (SD: $\pm 2.2 \mathrm{~dB}$ ), and the loud speech level was $69.5 \mathrm{~dB}$ (SD: $\pm 3.5 \mathrm{~dB}$ ). The formula for calculating HAI-v1 is expressed in Equation (1) below:

Gain $(\mathrm{dB})=$ MCL $(\mathrm{dB}$ HL $)+\mathrm{dB}$ HL to $\mathrm{dB}$ SPL correction factor - CSL (dB SPL) - occlusion effect $-6 \mathrm{cc}$ (headphone) and 2 cc coupler difference + adjustment values

In Eq. [1], target gains across different input level of sounds (soft, medium, and loud levels) were calculated by subtraction of the CSL from the MCL. Then, adjustment values across frequencies were added for the final insertion gain values. The adjustment values composed of a dynamic range adjustment value and a weighted factor based on the BIF for Korean (Jin et al., 2016; Lee et al., 2012). The dynamic range adjustment value refers to a weighted factor to reach the gain around the medium por- 
tion in the dynamic range of speech for soft, medium, and loud levels (Lee et al., 2012). Compared to the English BIF, low-frequency bands below $1,000 \mathrm{~Hz}$ were more important in the Korean BIF (Jin et al., 2016). Thus, enhanced low-frequency gains below 1,000 Hz were considered (Lee et al., 2012). The enhanced insertion gains were determined to reach the gain around the $2 / 3$ portion in the dynamic range of speech for soft, medium, and loud levels. The compression threshold of the HAI-vl was fixed at $51 \mathrm{~dB}$ SPL for all frequencies and the compression ratio was also fixed at each frequency band (e.g., 1.5:1 for $500 \mathrm{~Hz}, 1: 75: 1$ for $3,000 \mathrm{~Hz}$, and 1.3:1 for 4,000 Hz).

\section{Participants}

Twenty-four Korean listeners with mild-to-severe SNHL participated in this study. There were 19 male and 5 female participants (60-80 years). The mean pure-tone average thresholds for 250, 500, 1,000, 2,000, 4,000, and 8,000 Hz were 54.4, 54.2, 55.6, $59.8,71.2$, and $85.2 \mathrm{~dB}$ HL, respectively. Detailed subject information is described in Table 1. An instructor explained about this study to all participants and they read and signed a consent form before starting the evaluation.

\section{Procedure}

Three BTE type HAs were used for this study instead of participants' own HAs. The NAL-NL2 was applied to the Eleva 411 HA (Phonak, Stäfa, Switzerland), the DSL5.0 was applied to the Savia 311 (Phonak), and the HAI-v1 was applied to the prototype HA developed by Samsung Electronics (Yongin, Korea). Those HAs were randomly equipped to each participant's preferred ear. All testing was conducted in a sound attenuated booth.

For reception thresholds of sentences (RTSs), batteries of Korean hearing-in-noise test (K-HINT) in both quiet and in noise front conditions were conducted (Moon et al., 2008). For noise front testing, the noise was fixed at $65 \mathrm{~dB}$ A (babble noise). The criterion of the threshold in noise front was to find a signal-tonoise ratio (SNR) at which the subject could repeat sentences correctly at a rate of $50 \%$. To avoid learning effects, each participant listened to the same sentence only once. For both RTSs, the testing was conducted three times with different HA fitting formulae (HAI-v1, NAL-NL2, and DSL5.0) in a random order. The location of the speaker was 1 meter in front of the patient. When each test for a HA fitting formula was done, each participant was asked to indicate clarity among three choices (very clear, clear, and not

Table 1. Participant information for age, gender, tested ear, and hearing thresholds

\begin{tabular}{|c|c|c|c|c|c|c|c|c|c|}
\hline \multirow{2}{*}{ Subject number } & \multirow{2}{*}{ Age (years) } & \multirow{2}{*}{ Gender } & \multirow{2}{*}{ Ear } & \multicolumn{6}{|c|}{ Frequency in $\mathrm{Hz}$} \\
\hline & & & & 250 & 500 & 1,000 & 2,000 & 4,000 & 8,000 \\
\hline S1 & 77 & $\mathrm{M}$ & $\mathrm{R}$ & 55 & 45 & 45 & 65 & 85 & 105 \\
\hline S2 & 68 & $\mathrm{~F}$ & $\mathrm{~L}$ & 50 & 50 & 55 & 65 & 55 & 100 \\
\hline S3 & 74 & $\mathrm{~F}$ & $\mathrm{~L}$ & 60 & 55 & 55 & 55 & 60 & 75 \\
\hline S4 & 60 & M & $\mathrm{L}$ & 70 & 65 & 55 & 46 & 85 & 95 \\
\hline S5 & 76 & M & $\mathrm{R}$ & 60 & 50 & 45 & 60 & 70 & 80 \\
\hline S6 & 70 & M & $\mathrm{R}$ & 65 & 65 & 60 & 65 & 70 & 80 \\
\hline S7 & 78 & M & $\mathrm{L}$ & 30 & 50 & 55 & 60 & 60 & 75 \\
\hline S8 & 69 & M & $\mathrm{R}$ & 55 & 40 & 55 & 50 & 65 & 75 \\
\hline S9 & 74 & $\mathrm{~F}$ & $\mathrm{R}$ & 70 & 70 & 60 & 50 & 55 & 75 \\
\hline $\mathrm{S} 10$ & 77 & $\mathrm{M}$ & $\mathrm{R}$ & 45 & 50 & 65 & 65 & 70 & 60 \\
\hline $\mathrm{S} 11$ & 73 & M & $\mathrm{L}$ & 55 & 45 & 45 & 65 & 85 & 105 \\
\hline $\mathrm{S} 12$ & 71 & M & $\mathrm{R}$ & 40 & 55 & 55 & 45 & 75 & 85 \\
\hline S13 & 73 & M & $\mathrm{L}$ & 45 & 45 & 50 & 65 & 70 & 70 \\
\hline $\mathrm{S} 14$ & 69 & M & $\mathrm{R}$ & 35 & 40 & 55 & 75 & 65 & 90 \\
\hline S15 & 75 & M & $\mathrm{R}$ & 60 & 70 & 70 & 75 & 85 & 100 \\
\hline S16 & 79 & $\mathrm{M}$ & $\mathrm{R}$ & 60 & 65 & 65 & 45 & 75 & 95 \\
\hline S17 & 82 & M & $\mathrm{R}$ & 45 & 45 & 50 & 65 & 70 & 70 \\
\hline S18 & 79 & $\mathrm{M}$ & $\mathrm{R}$ & 60 & 60 & 55 & 60 & 60 & 75 \\
\hline S19 & 73 & $\mathrm{M}$ & $\mathrm{L}$ & 50 & 50 & 55 & 65 & 55 & 100 \\
\hline S20 & 73 & $\mathrm{~F}$ & $\mathrm{R}$ & 70 & 65 & 55 & 45 & 85 & 95 \\
\hline S21 & 80 & $\mathrm{M}$ & $\mathrm{R}$ & 80 & 80 & 70 & 65 & 90 & 85 \\
\hline S22 & 77 & $\mathrm{~F}$ & $\mathrm{~L}$ & 50 & 45 & 55 & 65 & 85 & 95 \\
\hline $\mathrm{S} 23$ & 57 & $\mathrm{M}$ & $\mathrm{L}$ & 55 & 55 & 55 & 65 & 65 & 85 \\
\hline $\mathrm{S} 24$ & 77 & M & $\mathrm{R}$ & 40 & 40 & 50 & 55 & 70 & 75 \\
\hline
\end{tabular}


clear). After completing all testing, participants were asked their overall preference among the three HA fitting formulae using a forced-choice procedure. Then, results of RTSs and preferences across HA fitting formulae were compared. Each participant took about 2 hours to complete all evaluations, with breaks
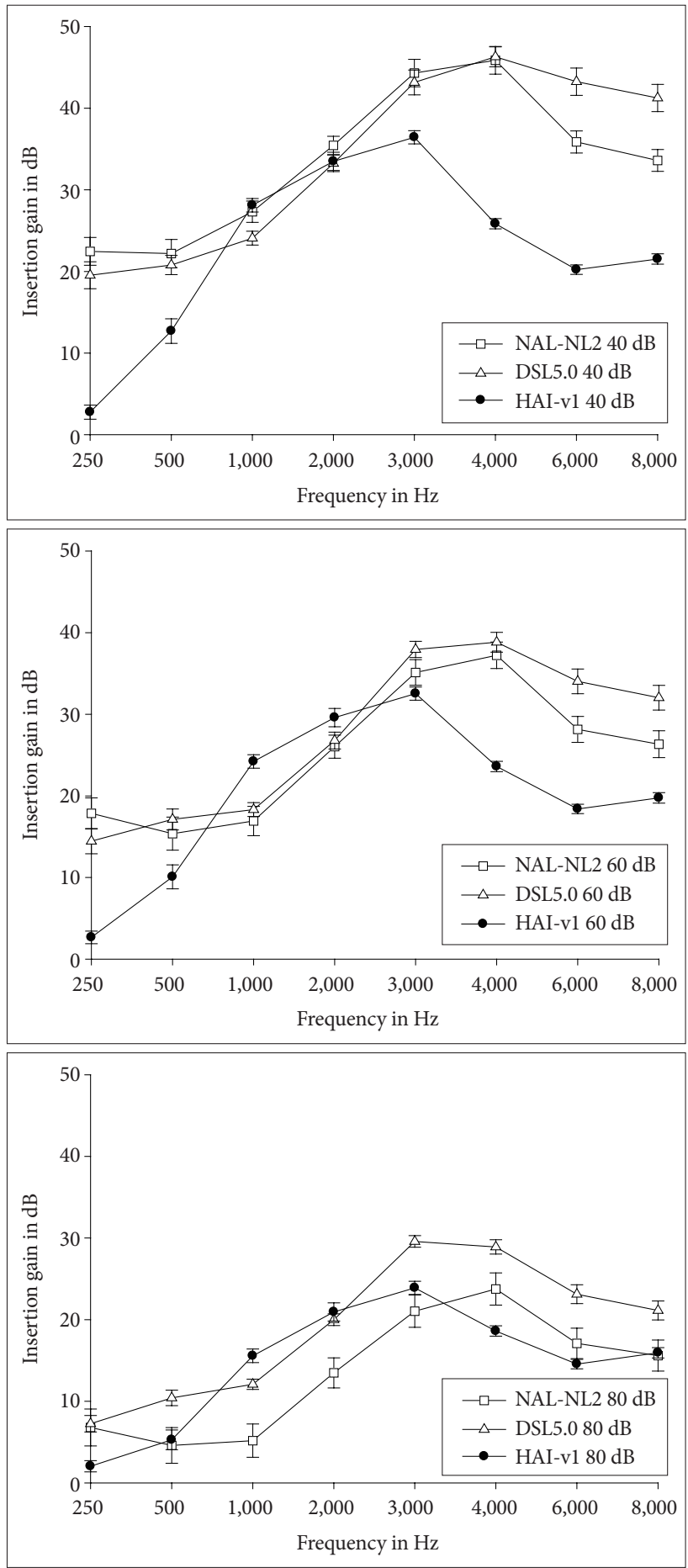

Figure 1. Insertion gains for soft ( $40 \mathrm{~dB} \mathrm{SPL})$, medium (60 dB SPL), and loud (80 dB SPL) levels across different HA fitting formulae [NAL-NL2 (square), DSL5.0 (triangle), and HAI-v1 (circle)]. HAI-v1: Hallym Audiology Institute-version 1. provided every 30 minutes.

\section{RESULTS}

As shown in Figure 1, insertion gains for soft (40 dB SPL), medium (60 dB SPL), and loud (80 dB SPL) sounds were different across different HA fitting formulae. For the soft level, insertion gains for NAL-NL2 and DSL5.0 were higher than the insertion gain for HAI-vl. For example, insertion gains for NAL-NL2 and DSL5.0 at the center frequency (CF) of $4,000 \mathrm{~Hz}$ were $45.87 \mathrm{~dB}$ and $43.16 \mathrm{~dB}$, respectively; but the insertion gain for HAI-vl was $25.85 \mathrm{~dB}$. For the medium and loud levels, insertion gains for HAI-vl at frequency areas between the CF of $1,000 \mathrm{~Hz}$ and the $\mathrm{CF}$ of $2,000 \mathrm{~Hz}$ were higher than insertion gains for other $\mathrm{HA}$ fitting formulae. For instance, the insertion gain of the medium level for HAI-vl at the $\mathrm{CF}$ of $1,000 \mathrm{~Hz}$ was $24.23 \mathrm{~dB}$, but insertion gains for NAL-NL2 and DSL5.0 were $16.92 \mathrm{~dB}$ and $18.33 \mathrm{~dB}$, respectively. However, insertion gains for NAL-NL2 and DSL5.0 at other frequency areas were higher than the insertion gain for HAI-vl.

Results of RTSs for the three HA fitting formulae are described in Table 2. In the quiet condition, mean RTSs for NALNL2, DSL5.0, and HAI-v1 were 60.2, 63.51, and $62.99 \mathrm{~dB}$ A, respectively. As the result of a univariate analysis of variance (ANOVA), there were no statistical differences among HA fitting formulae $(p=0.507)$. In the noise condition, mean RTSs for NALNL2, DSL5.0, and HAI-v1 were 10.03, 9.54, and $8.7 \mathrm{~dB}$ SNR, respectively. As the result of a univariate ANOVA, there were also no statistical differences among HA fitting formulae ( $p=0.659$ ).

Results of preferences are shown in Figure 2 (clarity) and 3 (overall preference). For clarity, more participants preferred sounds with HAI-v1 or NAL-NL2 than sounds with DSL5.0 (Figure 2). In the case of HAI-vl, 13 participants responded as 'very clear,' 5 participants responded as 'clear,' and 6 participants responded as 'not clear.' In the case of NAL-NL2, 13 participants responded as 'very clear,' 4 participants responded as 'clear,' and 7

Table 2. Mean RTSs in quiet and in noise conditions for NAL-NL2, DSL5.0, and HAl-v1

\begin{tabular}{lccc}
\hline \multicolumn{1}{c}{ RTS } & NAL-NL2 & DSL5.0 & HAI-v1 \\
\hline In quiet (dB HL) & & & \\
$\quad$ Mean & 60.20 & 63.51 & 62.99 \\
SD & 11.56 & 11.02 & 8.81 \\
In noise (dB SNR) & & & \\
$\quad$ Mean & 10.03 & 9.54 & 8.7 \\
SD & 5.31 & 5.43 & 4.21 \\
\hline
\end{tabular}

RTSs: reception thresholds of sentences, HAI-v1: Hallym Audiology Institute-version 1, SD: standard deviation, SNR: signal-to-noise ratio 


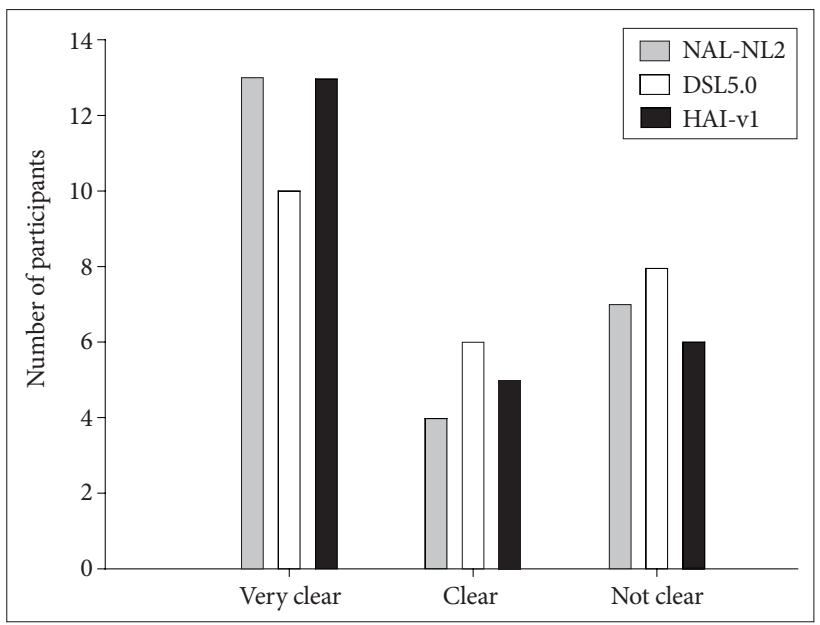

Figure 2. Results of clarity order of sounds across different HA fitting formulae as a function of degree of clarity ('very clear', 'clear', and 'not clear'). HAI-v1: Hallym Audiology Institute-version 1.

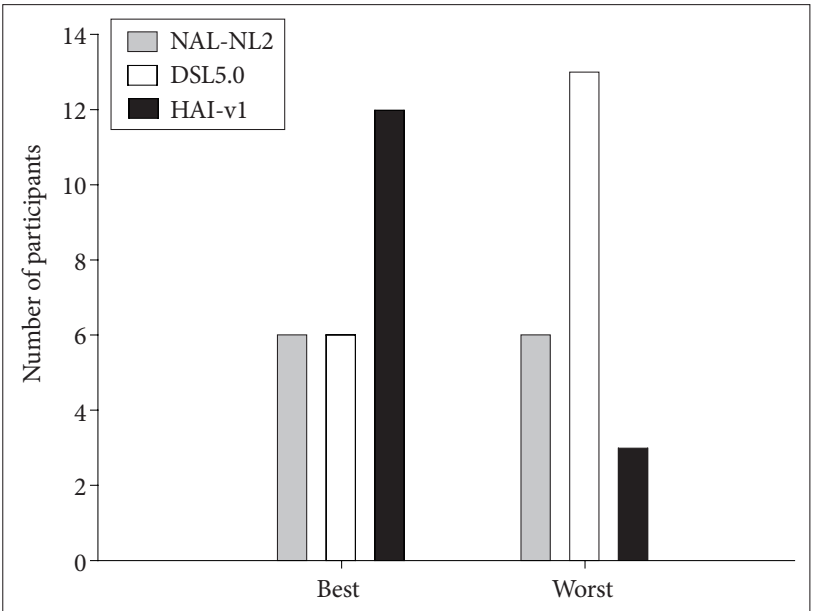

Figure 3. Results of overall preference order of sounds across different HA fitting formulae as a function of degree of preference ('best' and 'worst'). HAl-v1: Hallym Audiology Institute-version 1.

participants responded as 'not clear.' In the case of DSL5.0, 10 participants responded as 'very clear,' 6 participants responded as 'clear,' and 8 participants responded as 'not clear.' For the overall preference, more participants preferred sounds with HAI-v1 than sounds with NAL-NL2 or DSL5.0 (Figure 3). In the case of the HAI-vl, 12 participants responded as 'best,' and 3 participants responded as 'worst.' For the NAL-NL2, 6 participants responded as 'best,' and 6 participant responded as 'worst.' In the case of the DSL5.0, 6 participants responded as 'best,' and 13 participants responded as 'worst.'

\section{DISCUSSION}

The current study developed Korean language-specific HA fitting formula and compared speech perception with and without noise, clarity, and overall preference among amplified speech based on different HA fitting formulae such as the HAI-vl, NALNL2, and DSL5.0. The HAI-v1 was derived based on loudness growth for Koreans who have SNHL and the CSL for Koreans who have normal hearing. Although RTSs with and without noise were similar across the three HA fitting formulae, more participants preferred HAI-v1 than NAL-NL2 and DSL5.0 for clarity and overall preference.

Consideration of the language characteristics in HA fitting is not a new issue. When the NAL-NL2 was developed, tonal language was considered (Keidser et al., 2011). Because pitch variations are important for tone perception in tonal languages (e.g., Cantonese and Mandarin) compared to English, low frequency gains were enhanced when the NAL-NL2 for tonal language was developed (Gandour, 1981; Keidser et al., 2011).

However, the Korean language has different characteristics compared to English and tonal languages (Jin et al., 2014; Jin et al., 2016). Compared to English, the Korean language has different acoustic characteristics such as the LTASS, DR of speech, and the BIF which mentioned above (Jin et al., 2014; Jin et al., 2016; Noh \& Lee, 2012). Compared to tonal languages like Mandarin, the Korean language also has different acoustic characteristics like the BIF and the DR of speech. In terms of the BIF, the most important frequency areas for the Korean BIF were below $900 \mathrm{~Hz}$ and the cumulative importance weights were around $36 \%$ (Jin et al., 2016). However, the most important frequency areas for the Mandarin BIF were between $840 \mathrm{~Hz}$ and $1,370 \mathrm{~Hz}$ and the cumulative importance weights were around 29\% (Kuo, 2013). In terms of the DR of speech, the DR for Korean was narrower than the Mandarin DR in low-frequencies (less than the center frequency of $450 \mathrm{~Hz}$ ) (Jin et al., 2014). Based on these differences across languages, it seems difficult to say that the existing HA fitting formulae sufficiently reflects the acoustic characteristics of Korean.

The current study showed both limitations and possibilities for effects of a language-specific HA fitting formula. In the current study, RTSs with HAI-vl were not significantly different from RTSs with NAL-NL2 and DSL5.0. The RTS is a test to find the minimum intensity level $(\mathrm{dB})$ at which a listener can understand $50 \%$ of spoken sentences. Since this method can measure only the speech recognition at the threshold level, it seems to be less sensitive than the speech intelligibility test at various SNRs (Nilsson et al., 1994). If speech intelligibility performances in various SNRs are conducted, more specific comparisons for effects of HA fitting formulae will be possible. Moreover, the cur- 
rent study was conducted without adaptation periods of the HA with targeted fitting formulae. Because adaptation of the HA with a specific setting is important for speech perception and sound quality improvements, further studies should consider the adaptation period (Brooks, 1996). However, the current study partially showed the possibility that the HA fitting based on the Korean language may provide better HA satisfaction compared to the HA fitting formulae based on other languages. Thus, if more factors are considered to overcome limitations of the current study, effects of language-specific HA fitting formulae will be improved.

More limitations that can be improved are as follows. First, this study was conducted in a specific condition (e.g., adults with SNHL, monaural, experienced HA user, BTE HA, 2cc coupler, 8 channels). Thus, results of the current study could not reflect HA outcomes for various client characteristics, hearing loss, or device conditions. Second, the insertion gains for the HAI-vl were determined based on the loudness growth for Koreans who have SNHL and the CSL for Koreans who have normal hearing. However, other factors may also be considered to improve HA outcomes. For example, hearing loss-dependent compression can be additionally considered when insertion gains for the HAI-vl are determined. The current study used a fixed compression threshold for all frequencies and a fixed compression ratio for each frequency band. However, optimized compression conditions can be dependent on degrees of hearing loss (Keidser et al., 2011; Keidser et al.,2012).

Although there were several limitations for the current study, results of this study indicate that a HA fitting based on the Korean language may partially provide better HA outcomes compared to HA fitting formulae based on other languages. Thus, the current results may provide a baseline for developing HA fitting formulae based on Korean characteristics. If more factors are considered, more optimized HA fitting formulae may be developed for native Korean HA users.

\section{Acknowledgments}

This study was supported by a grant from Samsung Electronics Co., Ltd. and by Basic Science Research Program through the National Re- search Foundation (NRF) of Korea funded by the Ministry of Science, ICT \& Future Planning (NRF-2015R1C1A1A01052458).

\section{REFERENCES}

Brooks, D. N. (1996). The time course of adaptation to hearing aid use. British Journal of Audiology, 30(1), 55-62.

Gandour, J. (1981). Perceptual dimensions of tone: Evidence from Cantonese. Journal of Chinese Linguistics, 9(1), 20-36.

Jang, H. S., Lee, J. H., Lim, D. H., Lee, K. W., Jeon, A. R., \& Jung, E. J. (2008). Development of Korean standard sentence lists for sentence recognition tests. Audiology, 4(2), 161-177.

Jin, I. K., Kates, J. M., \& Arehart, K. H. (2014). Dynamic range for speech materials in Korean, English, and Mandarin: A cross-language comparison. Journal of Speech, Language, and Hearing Research, 57(5), 20242030.

Jin, I. K., Lee, J., Lee, K., Kim, J., Kim, D., Sohn, J., et al. (2016). The bandimportance function for the Korean standard sentence lists for adults. Journal of Audiology and Otology, 20(2), 80-84.

Keidser, G., Dillon, H., Carter, L., \& O’Brien, A. (2012). NAL-NL2 empirical adjustments. Trends in Amplification, 16(4), 211-223.

Keidser, G., Dillon, H., Flax, M., Ching, T., \& Brewer, S. (2011). The NALNL2 prescription procedure. Audiology Research, 1(1), 88-90.

Kuo, M. W. (2013). Frequency importance functions for words and sentences in Mandarin Chinese: Implications for hearing aid prescriptions in tonal languages. Sydney: University of Western Sydney.

Lee, J. H., Lee, K. W., Kim, J. S., Baik, H. J., \& Son, H. S. (2012). Development of a Clinical Protocol for Hearing Instruments. Final Report for the Research Project of Samsung Electronics. Suwon: Samsung Advanced Institute of Technology.

Moon, S. K., Kim, S. H., Mun, H. A., Jung, H. K., Lee, J. H., Choung, Y. H., et al. (2008). The Korean hearing in noise test. International Journal of Audiology, 47(6), 375-376.

Mueller, H. G., Hawkins, D. B., \& Northern, J. L. (1992). Probe Microphone Measurements: Hearing Aid Selection and Assessment. California, CA: Singular.

Nilsson, M., Soli, S. D., \& Sullivan, J. A. (1994). Development of the hearing in noise test for the measurement of speech reception thresholds in quiet and in noise. The Journal of the Acoustical Society of America, 95(2), 1085-1099.

Noh, H. \& Lee, D. H. (2012). Cross-language identification of long-term average speech spectra in Korean and English: Toward a better understanding of the quantitative difference between two languages. Ear and Hearing, 33(3), 441-443.

Palmer, C. V. \& Lindley IV, G. A. (2002). Overview and rationale for prescriptive formulas for linear and nonlinear hearing aids. In M. Valente (2nd ed). Strategies for Selecting and Verifying Hearing Aid Fittings. (pp. 1-22). New York, NY: Thieme Medical Publishers.

Park, Y. J. \& Lee, K. W. (2013). A changes of loudness growth as a function of hearing threshold level in adults with normal and sensorineural hearing loss. Audiology, 9(1), 25-32.

Scollie, S., Seewald, R., Cornelisse, L., Moodie, S., Bagatto, M., Laurnagaray, D., et al. (2005). The desired sensation level multistage input/output algorithm. Trends in Amplification, 9(4), 159-197. 